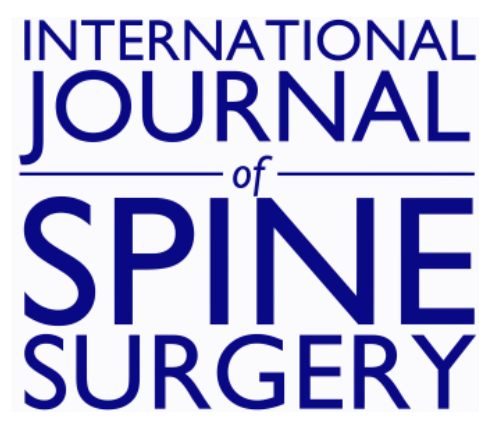

\title{
Five-Year Outcomes After Transforaminal Endoscopic Foraminotomy and Discectomy for Soft and Calcified Thoracic Disc Herniations
}

Karlo Houra, Robert Saftic and Martin Knight

Int J Spine Surg 2021, 15 (3) 494-503

doi: https://doi.org/10.14444/8071

http://ijssurgery.com/content/15/3/494

This information is current as of April 26, 2023.

Email Alerts Receive free email-alerts when new articles cite this article. Sign up at:

http://ijssurgery.com/alerts

The International Journal of Spine Surgery

2397 Waterbury Circle, Suite 1,

Aurora, IL 60504, Phone: +1-630-375-1432 


\title{
Five-Year Outcomes After Transforaminal Endoscopic Foraminotomy and Discectomy for Soft and Calcified Thoracic Disc Herniations
}

\author{
KARLO HOURA, MD, PHD, ${ }^{1,2}$ ROBERT SAFTIC, MD, MS, ${ }^{1}$ MARTIN KNIGHT, MD, FRCS MBBS ${ }^{3}$ \\ ${ }^{1}$ Aksis-Specialty Hospital for Spine and Orthopaedic Surgery, Petrovaradinska 1 Street, 10000 Zagreb, Croatia, ${ }^{2}$ University North, University Center Varazdin, \\ Jurja Krizanica Street 31b, 42000 Varazdin, Croatia, ${ }^{3}$ The Spinal Foundation, 17 Harley Street, London W1G 9QH
}

\begin{abstract}
Background: The aim of this study was to evaluate the safety and long-term clinical outcomes of transforaminal full endoscopic discectomy and foraminotomy performed with manual reamers under local anesthesia on soft and calcified herniated discs in the mid and lower thoracic spine.

Methods: Postoperative pain relief was self-evaluated by 16 patients using a visual analog scale (VAS) and Oswestry Disability Index (ODI). Patients were scored at 6, 12, 24, and 60 months after surgery.

Results: Significant pain reduction of more than $50 \%$ in the VAS score was achieved in 15 out of 16 patients at all review points throughout this study. Similarly, a decrease of more than 50\% in ODI scores was achieved in 15 out of 16 patients in all 4 review points. There were no surgical complications. Good postoperative results were achieved in patients regardless of the consistency of the disc herniation.

Conclusions: Transforaminal full endoscopic discectomy and foraminotomy with manual reamers performed under local anesthesia produces sustained reduction in pain and improves functionality in patients with mid and lower thoracic spine soft and calcified disc herniations. The surgery is safe and straightforward to perform with the correct training.

Level of Evidence: 4.

Clinical Relevance: Thoracic transforaminal endoscopic discectomy and foraminotomy, performed in TIVA, may be a useful adjunct for treatment of patients with soft and calcified disc herniations in thoracic spine.
\end{abstract}

Minimally Invasive Surgery

Keywords: thoracic spine, transforaminal endoscopy, hand reamers, disc herniation

\section{INTRODUCTION}

Anterior and nonanterior approaches have been used to access herniated discs in the thoracic spine. ${ }^{1-3}$ Nonanterior approaches are preferred because of reduced in-hospital morbidity and mortality rates. ${ }^{4,5}$ Recently, a minimally invasive transforaminal full endoscopic technique for the treatment of thoracic disc herniations is slowly being adopted. During endoscopy, manipulation of an already compromised thoracic spinal cord is kept to a minimum, potentially reducing postoperative morbidity and enhancing postoperative recovery.

There are few papers in the medical literature describing the removal of thoracic herniated discs using an entirely endoscopic transforaminal procedure. $^{4,6-13}$ Most reports address removal of soft thoracic disc herniations, and only 2 describe endoscopic removal of calcified thoracic disc pro- trusions endoscopically. ${ }^{6,10}$ Ten out of 16 patients $(62.5 \%)$ in our study had partially or totally calcified disc herniations, and 6 out of 16 patients $(37.5 \%)$ had soft herniated discs. Similar findings of calcified thoracic disc herniations were reported by Ruetten et al. ${ }^{6}$ In 2 patients with 2-disc herniations, both herniations were calcified. Final clinical outcomes after surgery were the same for all patients regardless of the consistency of the disc herniation. The only difference was slightly longer time to successfully complete the surgery when operating on calcified herniated discs since they were more adherent to the dura mater.

\section{METHODS}

\section{Patients}

From January 2008 to January 2015, 16 consecutive patients who had failed conservative medical 
therapy for thoracic back pain participated in this study. A single experienced neurosurgeon performed their examination and surgery. Approval of the hospital Ethics Committee was obtained, and all patients signed informed consent forms before enrollment in the study. Another experienced neurosurgeon, not directly involved in the surgery, conducted the outcome telephone reviews and collated the data for postoperative visual analog scale (VAS) and Oswestry Disability Index (ODI) scores.

\section{Procedure}

Preoperatively, all patients underwent clinical examination and magnetic resonance imaging (MRI) of the thoracic spine. Patients with calcified disc herniations also underwent preoperative computed tomography (CT) scans. All patients had symptoms for more than 6 months and a self-rated pain score of $5 / 10$ or more on a VAS. All patients had suffered failed conservative treatment including physical therapy, anti-inflammatory drugs, analgesics, and mild opioids as well as selective epidural transforaminal nerve root blocks. Transforaminal nerve root injections were used as diagnostic tools to identify painful thoracic disc, and all patients experienced temporary but marked pain relief after them. Discography was performed on all patients during surgery as a further means of confirming the painful disc. This procedure caused transient concordant pain in all patients. Ten patients had partially or totally calcified disc herniations, and 6 patients had soft herniated discs, and all were contained. Patients did not have deformity, instability, or prior surgery on the target segment. Patients who did not benefit from epidural nerve root blocks and patients who did not report concordant pain during discography were excluded from this study.

Before the surgery, a second experienced neurosurgeon, not directly involved in the surgery, evaluated patients for pain intensity using the VAS and ODI scores. During 4 different follow-up periods $(6,12,24$, and 60 months), the same neurosurgeon conducted a telephone interview rehearsing the same scoring tools.

\section{Demographic Features}

Over a period of 7 years, we prospectively treated 16 patients ( 10 men, 6 women; age ranged from 23 to 75 years). All patients had moderate to severe

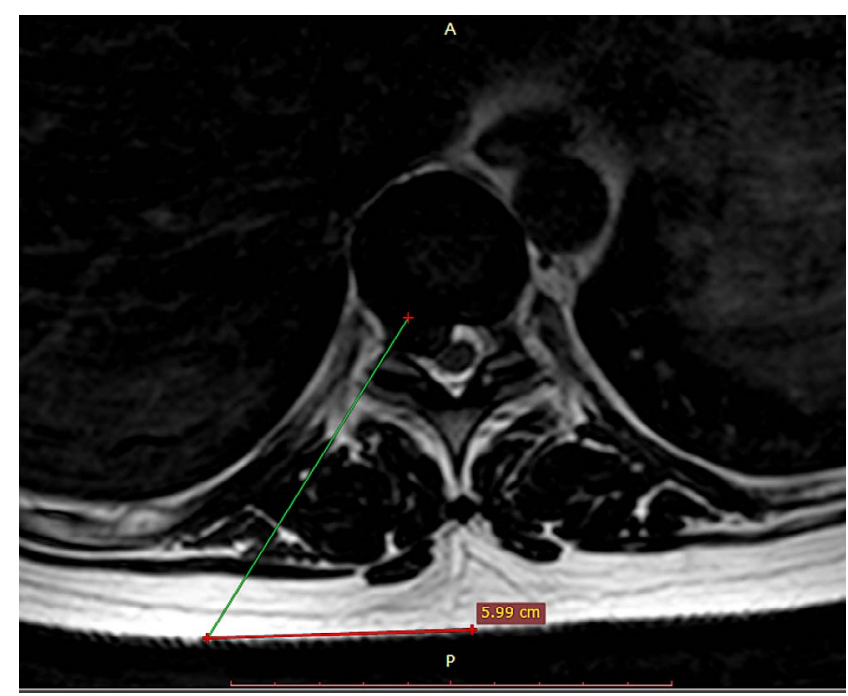

Figure 1. Determining skin entry point and a surgical trajectory using preoperative axial T2-weighted magnetic resonance imaging. The line is drawn from posterior annulus at the medial pedicular line to lateral margin of facet joint. The distance from the midline is $5.99 \mathrm{~cm}$.

predominantly thoracic pain without pain in the arms or legs. One patient had a disc herniation at the T6-7 level, 3 patients had disc herniations at the T8-9 level, 4 patients at the T10-11 level, 5 patients at the T11-12 level, and 1 patient had a disc herniation at the T12-L1 level. Two patients had disc herniations at both the T6-7 and T7-8 levels. Ten patients $(62.5 \%)$ in our study had calcified disc herniations, and 6 patients had soft herniated discs.

\section{Technique}

Using preoperative axial MRI, a desired trajectory was planned before the surgery for each individual patient. On the MRI scan, a skin entry point was determined by drawing a line from posterior annulus at the medial pedicular line, under the lateral margin of facet joint, and onto the skin (Figure 1). All patients were operated in a prone position using local anesthesia and total intravenous analgesia (TIVA) with biplane fluoroscopic guidance. ${ }^{14}$ After squaring the vertebral endplates in true anterior-posterior (AP) fluoroscopic view, the targeted intervertebral disc space was identified using a metal rod on the skin. The true x-ray AP view is achieved when the spinous process of the desired vertebra lies in the midline equidistant from both pedicles. Squaring the vertebral endplates determines optimal lateral access to the long axis of the desired disc and allows the surgeon the widest working space after endoscope placement. The skin entry point was measured from the midline on the 

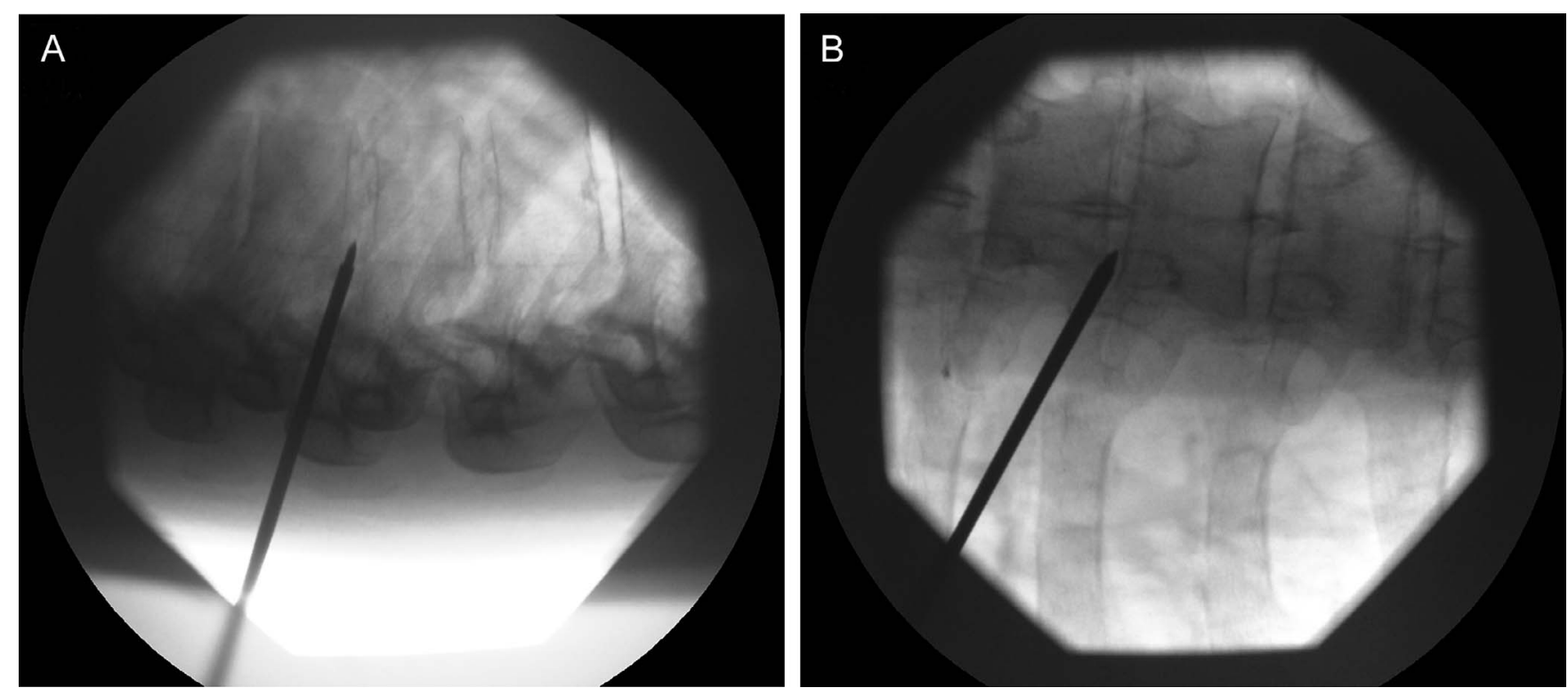

Figure 2. (A) Lateral intraoperative fluoroscopic view depicting final position of TOM Shidi bone needle in the posterior part of annulus fibrosus. (B) Anterior-posterior intraoperative fluoroscopic view of the TOM Shidi needle showing its tip in medial pedicular line. Vertebral endplates are squared.

side of the disc herniation, using the calculations from the presurgical planning. If the patient had a central, dorsomedial, or broad-based bilateral disc herniation, the approach side was that of the maximal pain. A $20 \mathrm{G}$ Spinal needle, $175 \mathrm{~mm}$ in length, was percutaneously advanced under fluoroscopic guidance to reach the desired intervertebral disc transforaminally. The tip of the needle was always directed toward the superior articular process of lower vertebra and thence the safe working zone medial to the exiting nerve. Once at the nucleus pulposus of the desired disc, discography was performed with $2 \mathrm{~mL}$ of Omnipaque 350 contrast and a couple of drops of indigo carmine blue dye. The purpose of indigo carmine dye was to color degenerated disc tissue blue to help the neurosurgeon intraoperatively distinguish degenerate herniated disc material from healthier disc tissue.

After discography and removal of the spinal needle, a cannulated TOM Shidi bone needle with a diamond tip was inserted through a $1 \mathrm{~cm}$ skin incision. Because the $20 \mathrm{G}$ spinal needle bends easily, it can be navigated into the disc for discography, but the trajectory is seldom optimal for the endoscopic procedure because it cannot be used to penetrate the bone of the superior articular process or, if necessary, rib head which is usually required to gain the desired working access. For this reason, the surgeon needs the bone piercing TOM Shidi needle which can be hammered through the superior articular process and, if necessary, the rib head to establish the desired trajectory for working cannula and endoscope. In the lateral view, the TOM Shidi needle was targeted toward the superior articular process of the lower vertebra and the rib head to access the desired foramen and intervertebral disc. In the final position, the tip of the TOM Shidi needle was located in the posterior annulus in the lateral view (Figure 2A) and at medial pedicle line in the AP view (Figure 2B). Extreme caution should be taken not to advance the TOM Shidi needle more medially because this could cause spinal cord injury. If the surgeon is concerned about the position of the sharp-tipped TOM Shidi needle, then this tip can be changed to a blunt-tipped version, and this needle can then be hammered deeper in the spinal canal, thus reducing the risk of neural damage. A Kirchner guide wire was then introduced through the TOM Shidi bone needle, and the trajectory for the endoscope to access the disc via the transforaminal route was established. This step of the surgery is of paramount importance because an unsatisfactory trajectory will prolong the surgery, increase $\mathrm{x}$-ray exposure, and in some cases, make it impossible to successfully complete the surgery. The paraspinal muscles were dilatated using tapered, cannulated dilators passed over the guide wire. Enlargement of the neural foramen was accomplished with manual reamers (Figure 3). By reaming the anterolateral margin of the superior articular process of the lower vertebra and anterolateral part of the rib head, the 

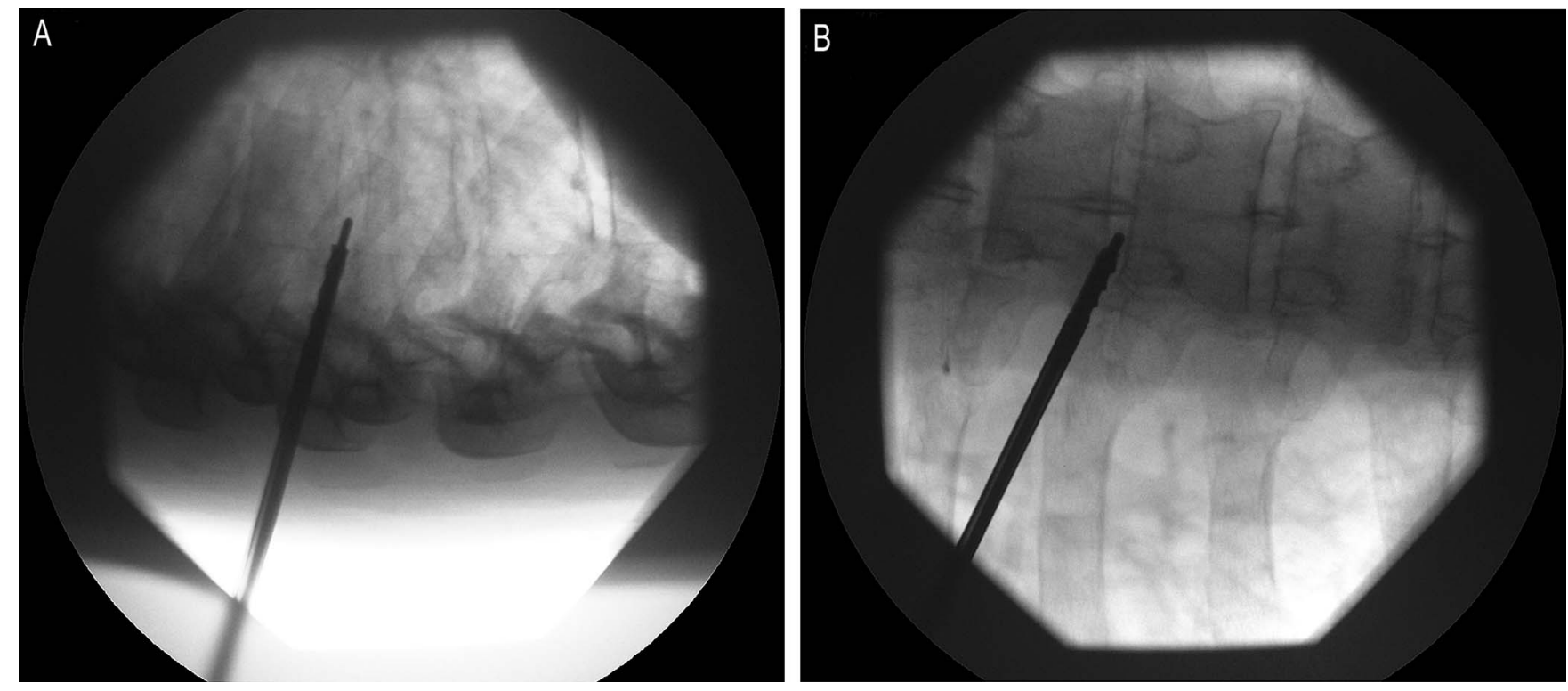

Figure 3. (A) Intraoperative fluoroscopic lateral view showing position of first manual reamer used for foraminotomy which is introduced over a guide wire. (B) Intraoperative fluoroscopic anterior-posterior view showing position of larger manual reamer with its tip at medial pedicle line at the entrance of the disk.

transforaminal route to the spinal canal was fashioned. The tip of the hand drills should not pass the medial border of the pedicle if injury to the spinal cord is to be prevented. All hand reamers were advanced over a guide wire under x-ray control. Operating on the patient under local anesthesia provides added protection against the risk of damaging the exiting nerve root or the spinal cord since the patient will alert the surgeon promptly if pain or discomfort is caused. Any minor discomfort caused replicates the predominant presenting symptoms, and this confirms that the correct level is being addressed. After foraminotomy, the hand drills were replaced by the muscle dilator, and the working cannula was railroaded to the foramen. A $4 \mathrm{~mm} 25^{\circ}$ angled working channel endoscope was then introduced through the working cannula. Once the endoscope was introduced, instruments like graspers, dissecting probes, drills, and even the endoscope were advanced under direct visual control beyond the medial pedicle line and even to the other side, as necessary. The combination of an angled field of view and the range of movement of the endoscope and working channel using a so-called "joystick technique" provided sufficient working area in all directions to enable exposure of the inferior and superior pedicle, intervertebral disc, ventral epidural space, and exiting nerve root. Displacement of the cannula and endoscope in various directions produces valuable small angular displacement in the working zone termed the "joystick technique," but forces should always be gentle, as excessive force on the endoscope may result in bending or damage to the optics. In partially or totally calcified disc herniations, a "bed" beneath the herniated portion of the disc should be performed first. This was done by drilling the posterior part of the vertebral body (Figure 4A). Soft disc from the intervertebral disc space under the calcified portion of the disc wall was then removed using graspers (Figure 4B). This maneuver is referred to as indirect decompression. ${ }^{6}$ After that, the calcified portion of the disc can be removed piecemeal while progressively detaching the wall from the dura using a blunt dissector (Figure 5). To avoid exaggerated pressure to the spinal cord during surgery, authors did not use a water pump. A saline container was elevated between $50 \mathrm{~cm}$ to $1 \mathrm{~m}$ above the patient. The height of the saline was adjusted to control intraoperative bleeding. Intraoperatively, restoration of pulsating dura mater was taken as direct evidence of sufficient surgical decompression.

The surgery usually takes between 60 and 80 minutes in experienced surgeons' hands and a little longer if the herniated disc is calcified. Patients were discharged home within 24 hours after the surgery.

\section{Statistical Analysis}

The VAS for pain and the ODI values were analyzed using mixed modeling. VAS or ODI were 

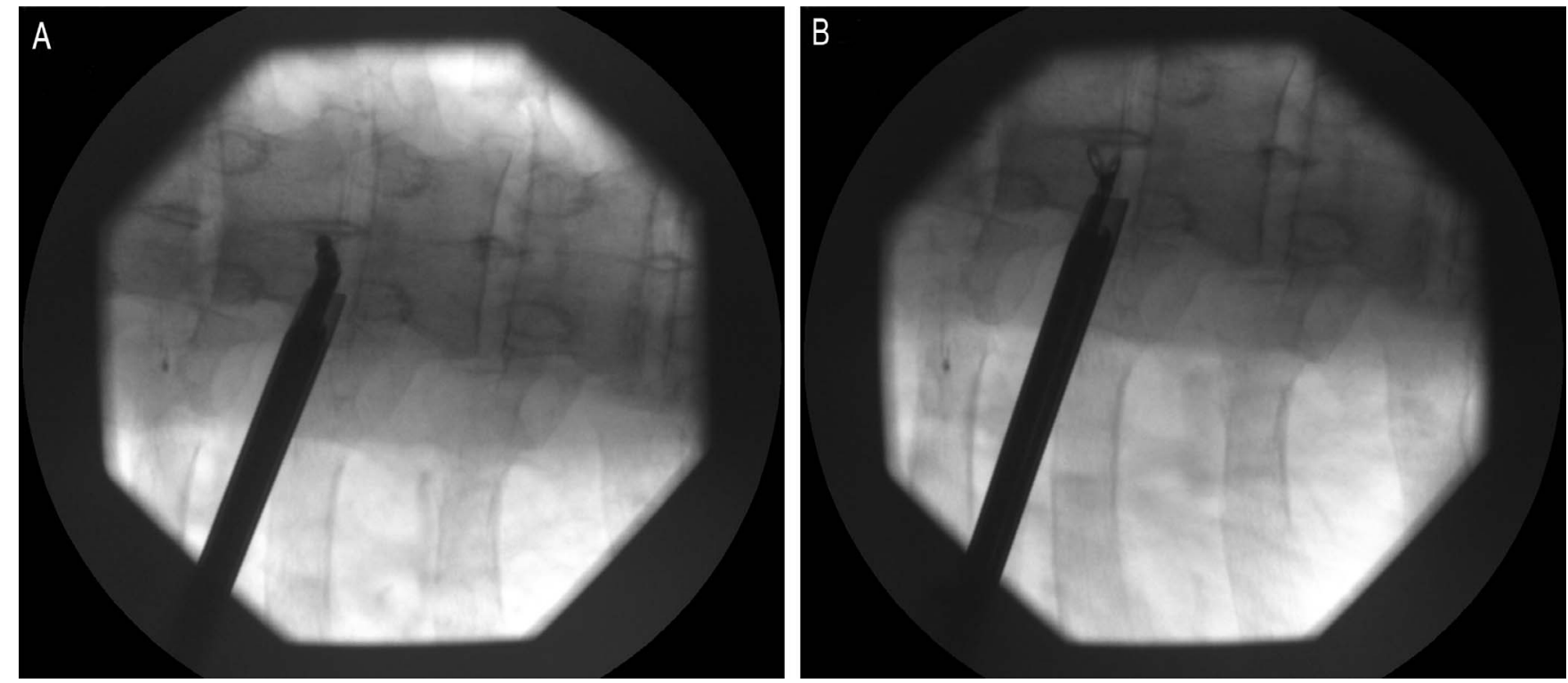

Figure 4. (A) Intraoperative fluoroscopic anterior-posterior (AP) view showing position of electric burr with curved tip used for bone drilling and making indirect decompression beneath the calcified portion of the disc. (B) Intraoperative fluoroscopic AP view showing position of grasper used for removal of soft disc.

defined as the dependent variable, while factors consistency of the disc herniation (with levels soft and calcified), timepoint (with levels pre and post) nested within consistency, and months (with levels 0 , $6,12,24$, and 60) nested within timepoint were set as fixed factors. Subject identifier was set as the random factor. Prior modeling, VAS and ODI were transformed by inverse transformation of ranks to standard normal distribution.

In total, 18 post hoc tests were applied (9 per index) to estimate the difference between calcified and soft disc herniation (1); after and before treatment for each consistency of the disc herniation (2); differences (after and before) between the calcified and soft disc herniation (1); and consecutive month levels in posttreatment period (12 versus 6, 24 versus 12, 60 versus 24) for each consistency of the disc herniation (6). False discovery rate was controlled using the Benjamini-Hochberg method, and only adjusted $P$ values were reported.

All statistical analyses were computed using $\mathrm{R}$ software for statistical computing, version 3.6.3. ( $\mathrm{R}$ Core Team, Vienna, Austria).
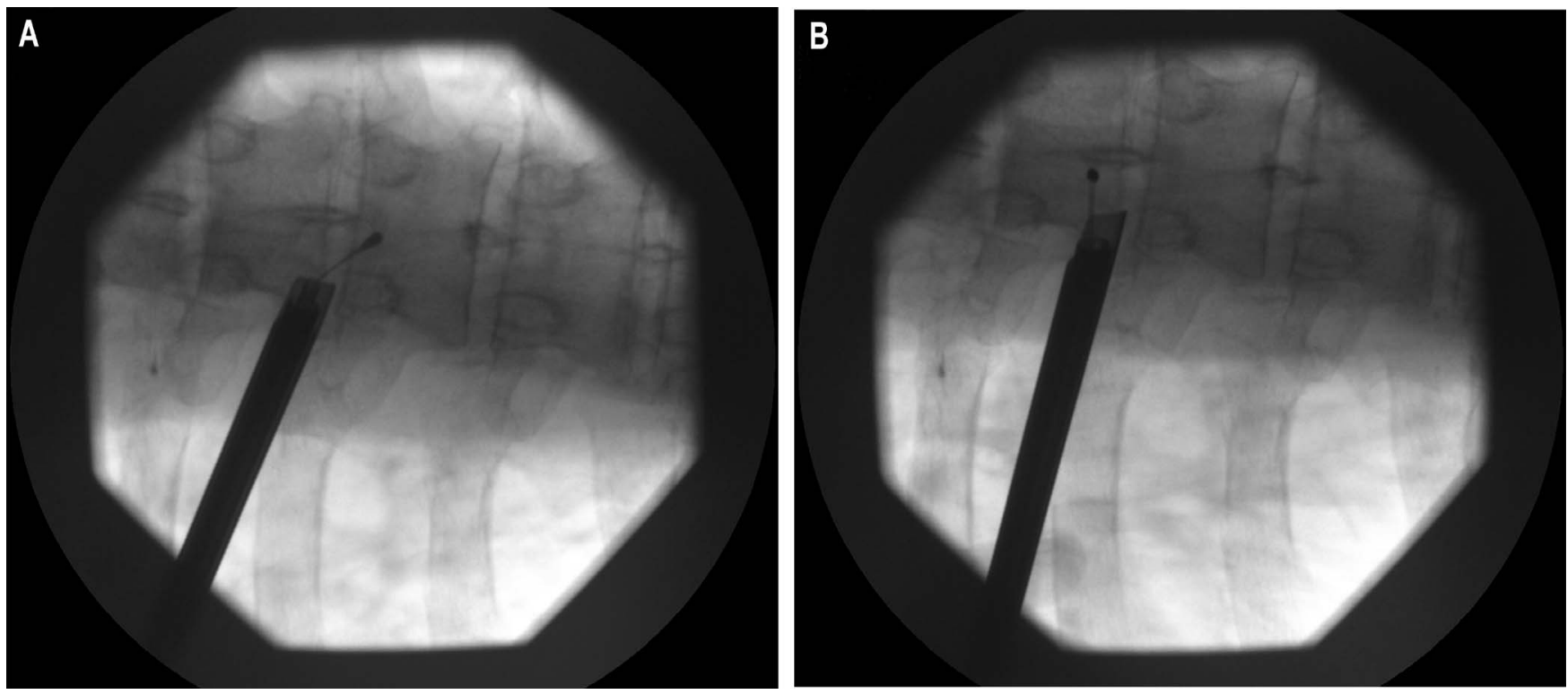

Figure 5. (A) and (B) Intraoperative fluoroscopic anterior-posterior view showing caudal and cranial position of blunt tip dissecting tool used while detaching the calcified herniation from the dura. 
Table 1. Preoperative and postoperative pain scores (in months) using visual analog scale (VAS) scores (0-10) with the level and consistency of the disc herniation for each patient in order of occurrence.

\begin{tabular}{|c|c|c|c|c|c|c|c|}
\hline Case No. & $\begin{array}{c}\text { Level of } \\
\text { Disc Herniation }\end{array}$ & $\begin{array}{l}\text { Consistency of } \\
\text { Disc Herniation }\end{array}$ & Pre-VAS & Post-VAS 6 & Post-VAS 12 & Post-VAS 24 & Post-VAS 60 \\
\hline 1 & T6-7 & Soft & 8 & 4 & 2 & 2 & 2 \\
\hline 2 & T8-9 & Soft & 7 & 3 & 1 & 1 & 1 \\
\hline 3 & T10-11 & Calcified & 10 & 2 & 1 & 1 & 1 \\
\hline 4 & $\mathrm{~T} 11-12$ & Soft & 7 & 3 & 2 & 1 & 1 \\
\hline 5 & T11-12 & Calcified & 9 & 4 & 3 & 3 & 3 \\
\hline 6 & T8-9 & Calcified & 10 & 4 & 2 & 2 & 2 \\
\hline 7 & T11-12 & Calcified & 8 & 4 & 4 & 4 & 4 \\
\hline 8 & T10-11 & Calcified & 9 & 3 & 2 & 1 & 1 \\
\hline 9 & T11-12 & Calcified & 9 & 3 & 1 & 1 & 1 \\
\hline 10 & T8-9 & Calcified & 8 & 4 & 3 & 3 & 3 \\
\hline 11 & T10-11 & Soft & 7 & 3 & 1 & 1 & 1 \\
\hline 12 & T11-12 & Soft & 6 & 1 & 0 & 0 & 0 \\
\hline 13 & T12-L1 & Soft & 8 & 2 & 1 & 1 & 1 \\
\hline 14 & T10-11 & Calcified & 9 & 4 & 3 & 3 & 3 \\
\hline 15 & T6-7 and T7-8 & Calcified & 8 & 3 & 1 & 1 & 1 \\
\hline 16 & T6-7 and T7-8 & Calcified & 9 & 7 & 6 & 6 & 6 \\
\hline
\end{tabular}

\section{RESULTS}

\section{Operative Findings}

Sufficient decompression was achieved in all cases. Intraoperatively, restoration of pulsating dura mater was taken as direct evidence of sufficient decompression during surgery. Postoperative MRI scans of the thoracic spine confirmed sufficient decompression had been achieved.

All patients tolerated endoscopic surgery well without serious postsurgical pain or neurological sequelae. During surgery, no complications occurred. One patient experienced transient numbness down both legs and trunk because of probable transient medulla concussion caused by hammering of the TOM Shidi bone needle while defining the trajectory. These symptoms resolved spontaneously after a couple of minutes, and the patient had an excellent outcome after the surgery.

\section{Survivability}

Where benefit was achieved after endoscopic surgery, it was sustained throughout the long follow up. Only 1 patient had a decrease of less than $50 \%$ on the ODI scale and had a poor VAS rating. He continued to have intermittent pain of moderate severity throughout the follow-up period. The exact reason for this outcome despite satisfactory postoperative hernia clearance on the CT scans is not understood. However, the fact that this patient underwent surgery for 2 large calcified disc herniations may be linked to a local cause.

\section{Analysis of Outcome Scores}

The pain intensity recorded for each patient using the VAS before and after endoscopic surgery at the defined follow-up periods is presented in Table 1 together with the level of the herniation and the consistency of the disc. Preoperative and postoperative ODI scores for each patient are presented in Table 2 together with the level of the herniation and the consistency of the disc. The median pain intensity (VAS) before surgery was 8 . At 6-month follow up, the median pain intensity was 3 ; at 12 months, it was 2; at 24- and 60-month follow up, median pain intensity was 1 . A similar trend was observed in ODI scores. The median ODI score was markedly reduced from 59 before the surgery to 13 or less in follow-up periods. Patients with calcified disc herniations had higher preoperative VAS scores and higher ODI scores.

Statistical analysis shown in Figure 6 demonstrates a significant difference in both VAS and ODI scores $(P<.001)$ before surgery against scores in each of 4 follow-up periods regardless of disc level or consistency of the disc herniation.

\section{Complications}

There were no cases of infection, dural tear, cord injury, or requirement for further surgery.

\section{DISCUSSION}

\section{Anesthesia During Surgery}

Ruetten et $\mathrm{al}^{6}$ used general anesthesia for endoscopic surgery. In our opinion and that of 
Table 2. Preoperative and postoperative Oswestry Disability Index (ODI) scores (0-100) in months with the level and consistency of the disc herniation for each patient in order of occurrence.

\begin{tabular}{|c|c|c|c|c|c|c|c|}
\hline Case No. & $\begin{array}{c}\text { Level of } \\
\text { Disc Herniation }\end{array}$ & $\begin{array}{l}\text { Consistency of } \\
\text { Disc Herniation }\end{array}$ & Pre-ODI & Post-ODI 6 & Post-ODI 12 & Post-ODI 24 & Post-ODI 60 \\
\hline 1 & T6-7 & Soft & 24 & 2 & 2 & 2 & 2 \\
\hline 2 & T8-9 & Soft & 18 & 6 & 6 & 6 & 6 \\
\hline 3 & $\mathrm{~T} 10-11$ & Calcified & 88 & 24 & 2 & 2 & 2 \\
\hline 4 & T11-12 & Soft & 16 & 4 & 4 & 4 & 4 \\
\hline 5 & $\mathrm{~T} 11-12$ & Calcified & 56 & 24 & 18 & 18 & 18 \\
\hline 6 & T8-9 & Calcified & 80 & 18 & 2 & 2 & 2 \\
\hline 7 & T11-12 & Calcified & 30 & 10 & 10 & 10 & 10 \\
\hline 8 & T10-11 & Calcified & 60 & 2 & 2 & 2 & 2 \\
\hline 9 & T11-12 & Calcified & 46 & 16 & 2 & 2 & 2 \\
\hline 10 & T8-9 & Calcified & 58 & 22 & 18 & 18 & 18 \\
\hline 11 & $\mathrm{~T} 10-11$ & Soft & 60 & 16 & 16 & 16 & 16 \\
\hline 12 & T11-12 & Soft & 30 & 2 & 2 & 2 & 2 \\
\hline 13 & T12-L1 & Soft & 62 & 4 & 4 & 4 & 4 \\
\hline 14 & T10-11 & Calcified & 70 & 24 & 22 & 22 & 22 \\
\hline 15 & T6-7 and T7-8 & Calcified & 66 & 6 & 2 & 2 & 2 \\
\hline 16 & T6-7 and T7-8 & Calcified & 80 & 66 & 60 & 60 & 60 \\
\hline
\end{tabular}

other authors, ${ }^{2,12}$ this is unnecessary and a rather risky approach, especially for inexperienced surgeons. Performing surgery under local anesthesia has several benefits. It avoids the potential compli-
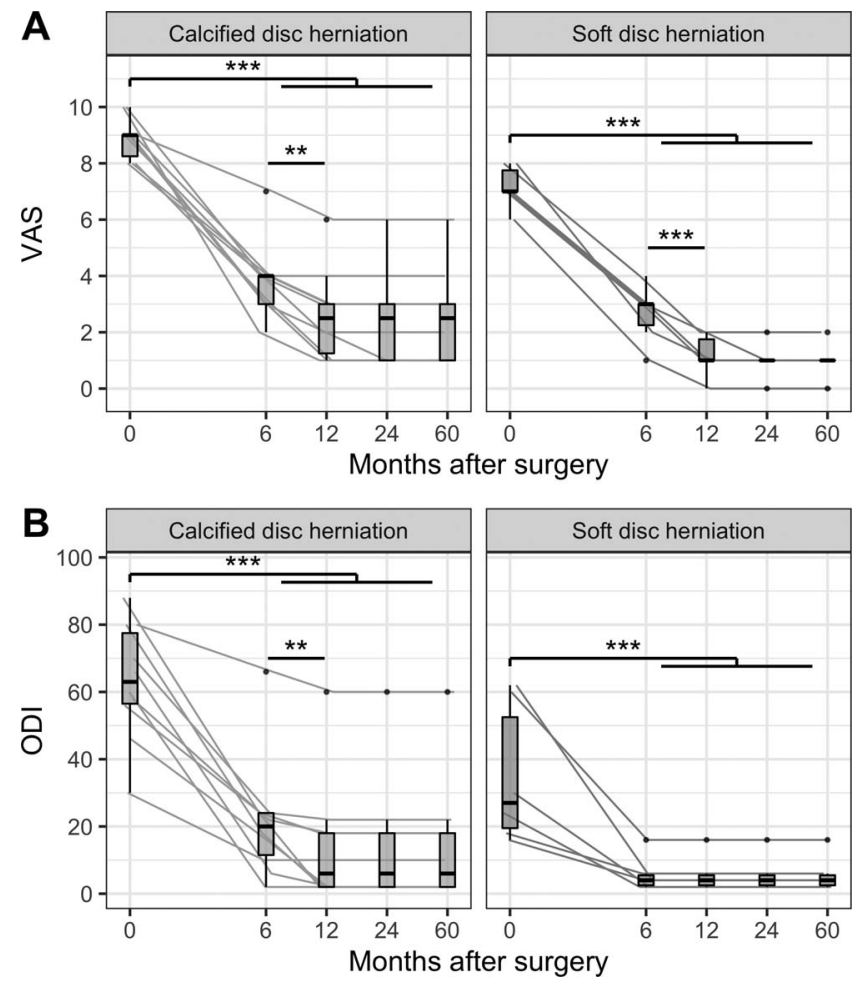

Figure 6. (A) The visual analog scale (VAS) for pain and (B) the Oswestry Disability Index (ODI) measurements in sampled timepoints. Measurements on the same subject were connected with lines. Measurements were also summarized with boxplots at each timepoint. Lower and upper box limits present first quartile $\left(Q_{1}\right)$ and third quartile $\left(Q_{3}\right)$, while thick line inside the box presents median. Whiskers are connecting minimum or maximum values within inner fence calculated as $1.5 \times$ interquartile range (calculated as $Q_{3}-Q_{1}$ ) below $Q_{1}$ or above $Q_{3}$, respectively. Any measurements which are not within inner fence are considered outliers and presented with points. Statistically significant differences (resulted from post hoc tests) are presented above compared groups with asterisks according to following $P$ value pattern: $.05>^{*}>.01>^{* *}$ $>.001>^{* * *}$. cations from endotracheal anesthesia and surgical trauma to the spinal cord. If the patient is operated upon under local anesthesia, there is no need for neuromonitoring. Furthermore, using local anesthesia avoids prolonged hospitalization resulting from the systemic effects of general anesthesia and facilitates quicker postoperative recovery.

\section{Surgical Technique}

In the treatment of calcified disc herniations, Gille et al ${ }^{13}$ proposed initial resection of the rib head and of the underlying vertebral pedicle to expose a healthy area of the dura. In our opinion, this procedure is not necessary, and thoracic transforaminal endoscopic discectomy and foraminotomy (TTEDF) can be successfully accomplished without significant rib head and pedicle resection if the correct approach trajectory for the endoscope is planned. This observation also corresponds to the conclusions of others. ${ }^{6,11}$ The combination of an angled field of view and freedom of movement of the endoscope using the so-called "joystick technique" results in adequate working area access. This technique preserves postoperative spinal stability and significantly reduces intraoperative blood loss and postoperative pain.

Calcified herniated discs can be successfully removed by drilling the "bed" beneath the calcified portion of herniation and by detaching the herniation from the posterior edge of the vertebral bodies. When a calcified portion of the herniated disc cannot be safely detached from the dura under direct vision in an "outside-in" technique, one should not insist on pursuing detachment. Aggressive maneuvers usually result in a dural tear or 
spinal cord injury. Instead, in these situations, the surgeon should make a "bed" beneath the attached part of the disc by drilling the bone of the posterior part of the endplate and the vertebral body. The soft part of the disc should then be removed. This is called indirect decompression. If the desired part of vertebra cannot be reached with the straight electric drill, one should use the flexible, angled electric drill. In the second step, the disc should be detached from the endplate and posterior longitudinal ligament using sharp dissection. Thus liberated, the disc wall attached to the dura can now float freely over the previously prepared "bed" and no longer compresses the spinal cord. This is called the "free-floating technique." Performing indirect decompression before the direct decompression for medial, calcified pathologies allows potentially dangerous manipulation of the attached dura and spinal cord to be avoided. When dissecting the calcified portion of the disc from dura, one should always start from the lateral part and progress to the medial part of attached disc wall. Dissection is performed using a curved, blunt tip probe with adjustable length. The maneuvers are slow and are carried in a craniocaudal direction.

\section{Choice of Approach}

Some authors advocate anterior approaches for medial calcified thoracic disc herniations. ${ }^{1,3}$ It is our opinion that using the transforaminal posterolateral approach is equally as efficient in treating these herniations and, by contrast, carries less risk of complications and enhances postoperative recovery. The feasibility and advantages stem from the fact that the posterior part of the thoracic vertebral body is rather concave, and only slight advancement with the endoscope and other working instruments under direct visual control can enable surgeon to easily reach the midline and, if necessary, to reach the contralateral side, a process enabled by the foraminotomy.

The limitation to this method is the fact that the learning curve of this technique takes a significant time to achieve maximum efficacy. This is due to the confined access arising from the bone anatomy, the vicinity of the pleura and the lungs, but also due to the small number of patients with symptomatic thoracic disc herniations who require surgery.

While many authors understand that full endoscopic transforaminal endoscopic thoracic discectomy is indicated for soft disc herniations, regardless of their location within spinal canal, our results indicate that the same technique can be equally used with hard disc herniations as well. The position within epidural space and foramen is irrelevant if the surgeon is familiar with thoracic vertebra anatomy and plans the optimal trajectory preoperatively. Hand reamers are used for the quicker entry procedure, and powered drills are reserved for use only when the position within the disc or in the spinal canal is secured and safe. All hand reamers have blunt tips, so if they are used gently, the instrument pushes and does not pierce the structures in front of it. Power drills are used exclusively under direct visual control.

The authors have no intention to present endoscopic thoracic discectomy as a mainstream technique. Instead, we wanted to present the evidence that this surgical technique can be very effective in such patients when used in experienced hands. The intention was to promote endoscopic surgery beyond the generally accepted boundaries and to encourage its wider use in centers currently responsible for treating thoracic disc protrusions.

\section{Duration of Follow Up}

This study is unique in the fact that the complete cohort patients reached 60 months of follow up. There is no other study in the published literature with a follow up this long achieved by all participants. The data presented in Tables 1 and 2 show 2 relevant features. Every patient who obtained at least $50 \%$ relief of pain using VAS also dramatically improved their ODI score. Patients with calcified disc herniations had higher preoperative ODI and VAS scores. There were no statistically significant differences between measured postoperative outcome and pain scores regardless of disc herniation consistency. There was only 1 patient who did not obtain at least $50 \%$ pain relief using VAS and ODI score.

Fifteen patients obtained at least $50 \%$ relief of pain over the 60 -month follow up. This provides a notional surgical success rate of $15 / 16(93.7 \%)$. This success rate is like the recently published work of Bae et al, ${ }^{12}$ but these authors only addressed soft thoracic disc herniations.

The sustained benefit achieved by TTEDF in this disabled group with failed conservative measures and a high incidence of calcified protrusions represents a significant success. It represents a valuable alternative to the conventional aggressive 
surgical approaches with prolonged hospital stay and greater damage to the bone and the soft tissue. These results correlate well with the shorter-term reports of other authors using the same endoscopic technique to treat herniated discs in thoracic spine and using the same measurement tools. ${ }^{4,6-9,11,12}$

\section{Analysis of a Poor Outcome}

One out of 16 patients $(6.25 \%)$ failed to achieve significant pain reduction using VAS score and decrease of ODI score at the end of the follow-up period of 60 months. This patient after surgery still had moderate thoracic pain. It is authors' opinion that this patient was unsatisfied after the surgery because he underwent endoscopic surgery at 2 levels for 2 giant calcified herniated discs and because the history of his pain before surgery was very long. This patient had a 4.5-year-long history of thoracic pain before surgery, and it is well known that patients with shorter duration of pain and less pronounced symptoms achieve better postoperative outcomes. We may only surmise that the pain generator in this patient was maybe located elsewhere and is still unidentified, that he had central or peripheral sensitization of his pain, or a local residual irritation of the dura or segmental nerves arising from tethering or ongoing degeneration within the disc.

Maintaining positive results in our patients over a long follow-up period after transforaminal endoscopic discectomy is in accord with the results of other authors ${ }^{2,7,10}$ whose positive results were unchanged during several months of follow up.

\section{Patient Safety}

Transforaminal endoscopic discectomy and foraminotomy is a safe surgical technique for the removal of both soft and calcified herniated discs in the mid and lower thoracic spine if performed under local anesthesia and conscious sedation (TIVA). Real-time patients' responses regarding pain or unwanted sensations prevent the surgeon from causing any serious damage to the nervous tissue during the procedure if the exact steps described in this paper are followed. No patients experienced infection, dural leaks, or permanent injury to the spinal cord, and in the follow-up period of 60 months, none of them required reoperation. The intraoperative blood loss was so low that it could not be measured due to the continuous irrigation.

\section{Study Strengths and Weaknesses}

Our study has several limitations. This was a prospective, observational, open-label study of a small sample of patients with no control group. Strengths included full cohort completion using 2 different measurement tools for the whole follow-up period of 60 months. Also, the continuity of patient review by an experienced neurosurgeon not directly involved in endoscopic surgery reduced the assessment bias.

\section{CONCLUSIONS}

Minimally invasive TTEDF in patients with soft and calcified disc herniations in the mid and lower thoracic spine with long-lasting back pain resistant to conservative treatment was effective in reducing pain and disability as measured by their VAS and ODI scores. Surgery under local anesthesia and TIVA with conscious sedation provided added safety to the procedure. The benefits were consistently sustained over a 5-year period, and the procedure was free of complications.

\section{REFERENCES}

1. Yoshihara H. Surgical treatment for thoracic disc herniation: an update. Spine (Phila Pa 1976). 2014;39(6):E406-E412.

2. Sharma SB, Kim JS. A review of minimally invasive surgical techniques for the management of thoracic disc herniations. Neurospine. 2019;16(1):24-33.

3. Bouthors C, Benzakour A, Court C. Surgical treatment of thoracic disc herniation: an overview. Int Orthop. 2019;43(4):807-816.

4. Ruetten S, Hahn P, Oezdemir S, et al. Full-endoscopic uniportal decompression in disc herniations and stenosis of the thoracic spine using the interlaminar, extraforaminal, or transthoracic retropleural approach. $J$ Neurosurg Spine. 2018;29(2):157-168.

5. Yoshihara H, Yoneoka D. Comparison of in-hospital morbidity and mortality rates between anterior and nonanterior approach procedures for thoracic disc herniation. Spine (Phila Pa 1976). 2014;39(12):E728-E733.

6. Ruetten S, Hahn P, Oezdemir S, Baraliakos X, Godolias G, Komp M. Operation of soft or calcified thoracic disc herniations in the full-endoscopic uniportal extraforaminal technique. Pain Physician. 2018;21(4):E331-E340.

7. Choi KY, Eun SS, Lee SH, Lee HY. Percutaneous endoscopic thoracic discectomy: transforaminal approach. Minim Invasive Neurosurg. 2010;53(1):25-28.

8. Nie HF, Liu KX. Endoscopic transforaminal thoracic foraminotomy and discectomy for the treatment of thoracic disc herniation. Minim Invasive Surg. 2013;2013:264105. doi:10. $1155 / 2013 / 264105$

9. Wagner R, Telfeian AE, Iprenburg M, et al. Transforaminal endoscopic foraminoplasty and discectomy for the 
treatment of a thoracic disc herniation. World Neurosurg. 2016;90:194-198.

10. Zhang LM, Lv WY, Cheng G, Wang DY, Zhang JN, Zhang XF. Percutaneous endoscopic decompression for calcified thoracic disc herniation using a novel $\mathrm{T}$ rigid bendable burr. Br J Neurosurg. 2019;28:1-3.

11. Bae J, Chachan S, Shin SH, Lee SH. Transforaminal endoscopic thoracic discectomy with foraminoplasty for the treatment of thoracic disc herniation. J Spine Surg. 2020;6(2):397-404. doi:10.21037/jss.2019.11.19

12. Bae J, Chachan S, Shin S, Lee S. Percutaneous endoscopic thoracic discectomy in the upper and midthoracic spine: a technical note. Neurospine. 2019;16(1):148-153.

13. Gille O, Soderlund C, Razafimahandri HJ, Mangione PF, Vital JM. Analysis of hard thoracic herniated discs: review of 18 cases operated by thoracoscopy. Eur Spine J. 2005;15(5):537-542.

14. Knight MTN, Krishnan A. Spinal surgery. In: Whitwam JG, McLoy RF, eds. Principles and Practice of Sedation. Oxford: Blackwell Science; 1998:178-181.

Disclosures and COI: No funding was received for this research. All authors certify that they have no affiliations with or involvement in any organization or entity with any financial interest (such as honoraria; educational grants; participation in speakers' bureaus; membership, employment, consultancies, stock ownership, or other equity interest; and expert testimony or patentlicensing arrangements), or nonfinancial interest (such as personal or professional relationships, affiliations, knowledge, or beliefs) in the subject matter or materials discussed in this manuscript. All procedures performed in our study involving human participants were in accordance with the ethical standards of the institutional research committee (Aksis - Specialty Hospital for Spine and Orthopedic Surgery) and with the 1964 Helsinki Declaration and its later amendments or comparable ethical standards. Informed consent was obtained from all individual participants included in the study. All data analyzed during this study are included in this published article.

Corresponding Author: Karlo Houra, MD, $\mathrm{PhD}$, Aksis-Specialty Hospital for Spine and Orthopedic Surgery, Petrovaradinska 1, 10000 Zagreb, Croatia. Phone: +385 91 5421916; Fax: +385 13877 414; Email: khoura@unin.hr

Published 1 June 2021

This manuscript is generously published free of charge by ISASS, the International Society for the Advancement of Spine Surgery. Copyright $\odot 2021$ ISASS. To see more or order reprints or permissions, see http://ijssurgery.com. 